\title{
Covid-19: Delta infections threaten herd immunity vaccine strategy
}

\section{Owen Dyer}

Epidemiologists are adjusting their expectations for the future course of the pandemic after data from a recent outbreak in Massachusetts in the US suggest that while vaccination remains highly protective against the worst consequences of infection, it may not be sufficient on its own to stop the spread of the delta variant.

Testing conducted among Massachusetts residents during an outbreak in Provincetown, a popular weekend getaway spot, from 3 to 17 July found that $75 \%$ of those infected were fully vaccinated, in a state where $69 \%$ of adults were fully vaccinated. ${ }^{1}$

Among the vaccinated with breakthrough infections, the difficulty of detecting virus in the nasal passages, known as the cycle threshold value, was almost identical to that seen in the unvaccinated. This finding suggests that both groups carried equal viral loads and were equally likely to pass on their infections, the US Centers for Disease Control and Prevention (CDC) warned.

The results support claims that vaccinated people are playing a role in the summer surge in delta variant infections and led the CDC to reinstate its recommendation that vaccinated people wear masks indoors.

The CDC published the Provincetown data on 30 July, but a slide presentation mentioning the findings on viral load was obtained in advance by the Washington Post. ${ }^{2}$ Epidemiologists who were shown these findings told the newspaper that they changed the calculus going forward. "I finished reading it significantly more concerned than when I began," said Robert Wachter, chairman of the Department of Medicine at the University of California at San Francisco.

Jeffrey Shaman, an epidemiologist at Columbia University, said, "In some sense, vaccination is now about personal protection-protecting oneself against severe disease. Herd immunity is not relevant as we are seeing plenty of evidence of repeat and breakthrough infections."

Andrew Noymer, an epidemiologist at the University of California at Irvine, said, "Although most cases did not result in hospital admission, showing the vaccine works in an important way, this study is portentous for the achievement of herd immunity. If the vaccinated can become infected and, we believe from other studies, potentially spread covid, then herd immunity becomes more mirage than oasis."

CDC director Rochelle Walensky said in a statement, "This finding is concerning and was a factor leading to CDC's updated mask recommendation.” The delta variant is at least twice as contagious as the original SARS-Cov2 virus, she said.
The CDC slide presentation described the delta variant as more infectious than the common cold and as infectious as chickenpox. It also pointed to studies from Canada, Scotland, and Singapore suggesting that delta infection is about twice as likely as ancestral strains to result in pneumonia or hospital admission.

Of 346 vaccinated people who tested positive in the Provincetown outbreak, $79 \%$ were symptomatic, and sample genome testing suggested that $90 \%$ had the delta variant. Four were admitted to hospital and none died.

The vaccine continues to offer strong protection against severe disease. There are currently about 35 ooo symptomatic infections a week among 162 million vaccinated Americans, the CDC slide presentation said. The vaccine brings a threefold reduction in risk of infection, an eightfold reduction in risk of symptomatic disease, and a 25 -fold reduction in risk of hospital admission or death, the agency estimates. The CDC had learnt by last week of 6239 hospital admissions and 1263 deaths among the fully vaccinated-a death rate of $0.0008 \% .^{3}$

The CDC must improve the public's understanding of breakthrough infections, the agency's leaked slide presentation said, using personal stories and comparisons to rates of poor outcomes in the unvaccinated. It should stress vaccination's high impact on death and hospital admission rates but make clear that vaccine effectiveness against infection is lower, the document said, urging the CDC to "acknowledge that the war has changed."

\section{UK experts predict new variants will evade vaccines}

In the UK the expert advisory board SAGE predicted that new variants will eventually overcome existing vaccines. ${ }^{4}$

"As eradication of SARS-CoV-2 will be unlikely, we have high confidence in stating that there will always be variants," the panel reported. A variant bringing more severe disease with mortality like SARS-CoV (10\%) or MERS-CoV ( 35\%) was a "realistic possibility,” as was a variant that finds an animal reservoir then returns to strike humans in an evolved, vaccine resistant form. A sharp antigenic shift that changes the spike protein on the virus enough to evade current vaccines is also a realistic possibility, SAGE said.

A slower antigenic drift that "eventually leads to current vaccine failure" is "almost certain," SAGE warned. Meanwhile the main hope for an end to the pandemic, that the virus will remain highly transmissible but become less harmful, "like the human coronaviruses that cause common colds," 
was rated a realistic possibility in the long term but "unlikely in the short term.”

1 Brown CM, Vostok J, Johnson H, etal. Outbreak of SARS-CoV-2 infections, including covid-19 vaccine breakthrough infections, associated with large public gatherings: Barnstable County, Massachusetts, July 2021. Morbidity and Mortality Weekly Report. 2021. 30 July 2021. www.cdc.gov/mmwr/volumes/70/wr/mm7031e2.htmdoi: 10.15585/mmwr.mm7031e2.

2 Read: Internal CDC document on breakthrough infections. Washington Post. 30 July 2021. www.washingtonpost.com/context/cdc-breakthrough-infections/94390e3a-5e45-44a5-ac40$2744 \mathrm{e} 4 \mathrm{e} 25 \mathrm{f} 2 \mathrm{e}$.

3 National Center for Immunization and Respiratory Diseases. Covid-19 vaccine breakthrough case investigation and reporting. 26 July 2021. www.cdc.gov/vaccines/covid-19/health-departments/breakthrough-cases.html.

4 Scientific Advisory Group for Emergencies. Long term evolution of SARS-CoV-2, 26 July 2021. 30 July 2021. https://assets.publishing.service.gov.uk/government/uploads/system/uploads/attachment_data/file/1007566/S1335_Long_term_evolution_of_SARS-CoV-2.pdf.

This article is made freely available for use in accordance with BMJ's website terms and conditions for the duration of the covid-19 pandemic or until otherwise determined by BMJ. You may use, download and print the article for any lawful, non-commercial purpose (including text and data mining) provided that all copyright notices and trade marks are retained. 